\title{
Taeniothrips zurstrasseni (Thysanoptera, Thripidae), a new thrips species found in Poland
}

\author{
Irena ZAWIRSKA \\ Institute of Plant Protection, PAS, Miczurina 20, 60-318 Poznan, Poland; \\ address for correspondence: Sewerynów $4 m$ 4b, 00-331 Warszawa
}

\begin{abstract}
Taeniothrips zurstrasseni, a new species found in the damp environments of the northern Poland, is described and figured. Imagines and larvae were collected from flowers of various plants (most frequently from Stachys palustris) in summer, mainly in July and August.
\end{abstract}

Key words: Insecta, Thysanoptera, Thripidae, Taeniothrips, new species, north Poland

\section{INTRODUCTION}

Thripidae is the largest family of the order Thysanoptera in Polish fauna, with 137 species belonging to 46 genera recorded to date (Kucharczyk 2007). Three species of the genus Taeniothrips Amyot \& Serville, 1843 have been known from Europe so far: Taeniothrips picipes (Zetterstedt, 1828), T. inconsequens (Uzel, 1898), and T. arbutti Bournier, 1983 (zur Strassen, 2003). T. picipes and T. inconsequens are distributed in the Palaearctic region, $T$. arbutti has been hitherto noted in south France, Spain and Morocco. T. picipes and $T$. inconsequens can be found all across Poland; both species are polyphagous - T. picipes lives on flowers of many plants, $T$. inconsequens - on spring flowers, mainly on the blossom of trees and shrubs, in particular Rosaceae.

The specimens of Taeniothrips zurstrasseni sp. nov. were found on the territory of Slowiński National Park, which is located in the central part of the Polish Baltic Coast, in a region with a relatively mild and windy climate characterized by mild winters, not very hot summers, a rather short growing period, with high relative humidity but low annual precipitation. The flora of the Park represents different habitats and geographical elements. Besides plants common to the whole of central Europe, there are a number of plants typical of the boreal, Atlantic and oceanic climate (Piotrowska, 1997).

\section{METHODS}

Samples of thrips were collected from dune, forest, peat and meadow plants many times in the years 1991-2001. The specimens of the new species were found for the first time on a blooming Dactylorhiza maculata on 1 July 1998. In the following years, Dactylorhiza maculata plants were not found at all; the specimens of the new species were found and collected only in the year 2001 .

Two methods were used to collect the insects: specimens were either captured directly from plants into an alcohol solution or collected by means of a sweep net. The specimens of Taeniothrips zurstrasseni were collected from blooming plants growing in two damp sites in the middle part of Slowiński National Park (SNP) - alongside a drain channel (Long Channel) near Dolgie Wielkie Lake, and on a peat meadow with Juncus effusus and a peat meadow with various flowering plants near Gardno Lake. 

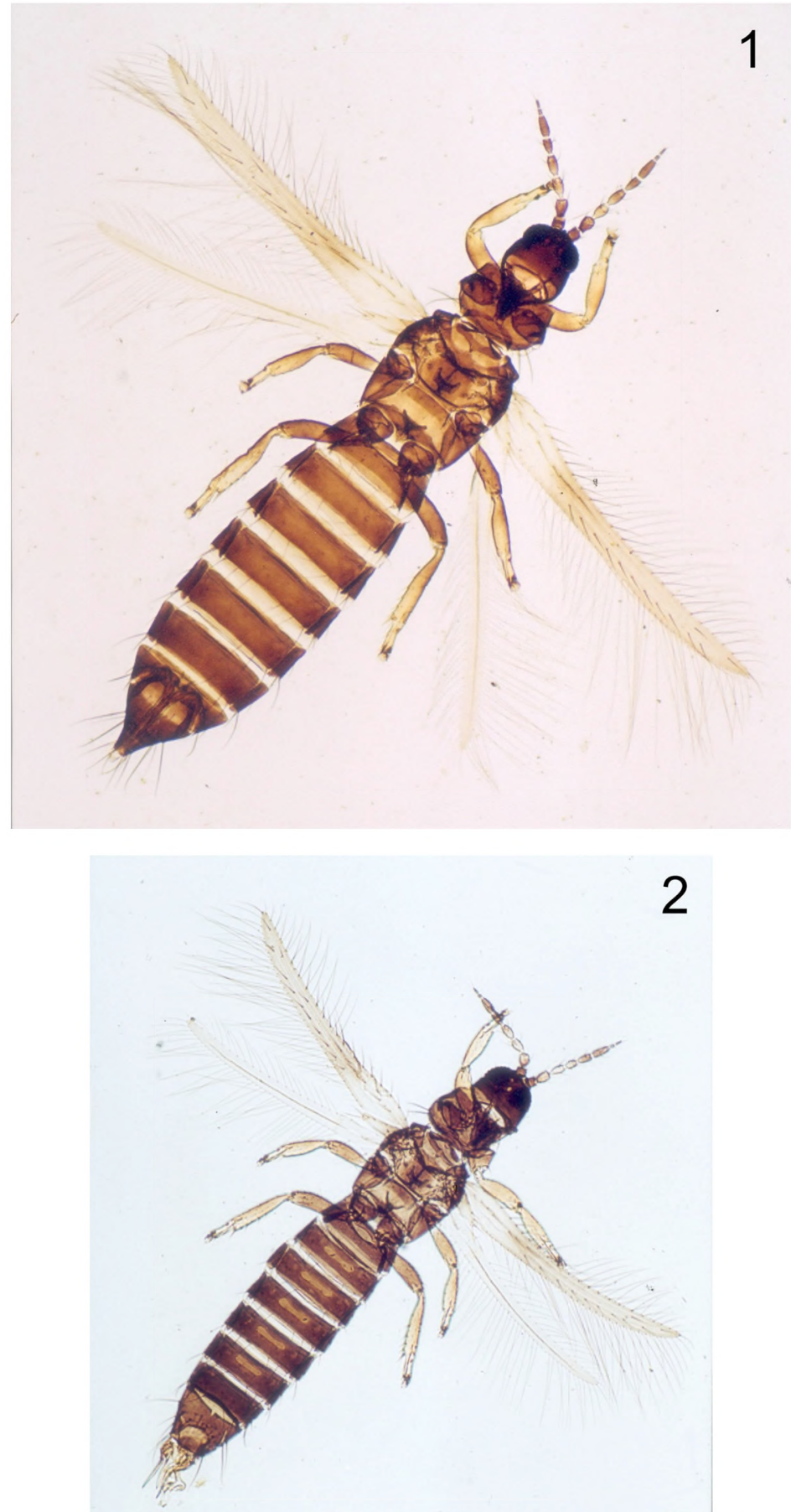

Fig. 1-2. Taeniothrips zurstrasseni sp. nov., paratypes: 9.08.2001, on by Long Channel in Słowiński National Park, collected on Stachys palustris; 1 - habitus of female, 2 - habitus of male; both ventral view (photo W. Sierka) 
RESULTS

Taeniothrips zurstrasseni sp. nov.

(Figs 1-11)

Type material: Holotype: $q$ found 3.08.2001 on a flowering Stachys palustris by Long Channel in SPN, leg. I. Zawirska.

Paratypes found in samples of different plants, all in SPN: 5 q, 1 ठै, 1.07 .1998 on Dactylorhiza maculata by Long Channel; 1 , 7.07.2001 on Lysimachia vulgaris by Long Channel; $5 q, 3 \hat{\partial}, 29.07 .2001$ on Stachys palustris by Long Channel; 1 q, $2 \hat{\partial}, 2.08 .2001$ on peat meadow near Gardno Lake; $69,2 \AA, 2$ larvae, 3.08.2001 on Stachys palustris by Long Channel; 3 , 5 o, 1 larva, 9.08.2001 on Linaria vulgaris by Long Channel; 1 , , 5 larvae, 27.08.2001 on Stachys palustris by Long Channel; 1 q, 2 larvae 29.08.2001 on Stachys palustris on peat meadow near Gardno Lake. All specimens leg. I. Zawirska.

The holotype and some paratypes of the species have been deposited in the collection of the Department of Zoology of Maria Curie-Sklodowska University in Lublin. 1 $\hat{\partial}, 1$ t and one larva have been deposited in the Museum and Institute Zoology PAS in Warsaw.

Material examined: $16 q, 1 \hat{\delta}, 1.07 .1998$, on blooming Dactylorhiza maculata; 2 , 7.07.2001, on inflorescences of Lysimachia vulgaris; 10 . , 3 है, 1 larva, 29.07.2001, on

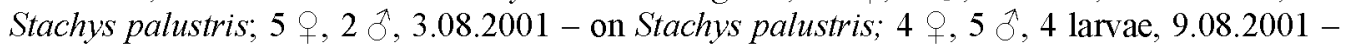
on Stachys palustris; 13 +, 1 ภ, 21 larvae, 27.08.2001 - on Stachys palustris; 2 , , 3.09.2001 on Stachys palustris; all found by Long Channel in SPN; $1 q$ on Lythrum salicaria and $6 q+$ $4 \hat{\sigma}$ on Stachys palustris, 2.08.2001, peat meadow near Gardno Lake; 1q, 2 larvae on Lysimachia vulgaris, 3.09.2001, same place; all specimens leg. I. Zawirska.

Etymology. I dedicate this new species to Dr. Richard zur Strassen, a great authority on the taxonomy of Thysanoptera.

Diagnosis. Species of medium size, female and male dark (Fig. $1 \& 2$ ). Head with slightly convex sides of eyes and cheeks. Antennae relatively short. Medial segments rather thick-set, segment III of similar length as segments IV and VI, segment V usually almost twice as long as wide, stylus relatively long. Upper vein of forewing with 3 or 2 distal setae. Setae S1 on metanotum located closer to the middle of sclerite, at a distance from fore margin; pair of campaniform sensilla absent, seldom one sensillum present. Setae S1 on abdominal tergite I longer and stronger than on tergites II-V. Dorsal split on segment X usually absent. In males both canaliculi of aedeagus with strong thorn in distal part.

Larva II white-grey with dark spots on head and thorax, antennal stylus strikingly dark, dark band on hind parts of abdominal segments IX and X, and often with light red internal pigment spread all over the body. Antennal segment III without microtrichia. Hind margin of segment IX without comb. The sculpture is very subtle, plaques nearly flat in places, often without microtrichia, and sometimes without plaques. Setae rather long and slightly darkened, dorsal - strong and blunt at end, ventral - subtler and sharp at end.

\section{Description}

Female $1500-1840 \mu \mathrm{m}$ long, body brown, fore legs and all tibiae slightly paler, especially in distal parts; all tarsi yellow. Antenna dark, only the apex of segments II, III and base of IV paler. Except light base part, forewing rather dark, beige-grey, darkest in region of vein bifurcation; also clavus partly darkened. Hindwing regularly light yellow-beige. Body and wing setae rather darkened, ovipositor yellowish.

Head (Fig. 3) 110-127 $\mu \mathrm{m}$ long, width in eyes 142-169 $\mu \mathrm{m}$, behind eyes 137-165 $\mu \mathrm{m}$, in cheeks 157-182 $\mu \mathrm{m}$, maximally (in cheeks) 1.46-1.62 times wider than longer. Compound eye $60-75 \mu \mathrm{m}$ long, occupies $56.8-65.2 \%$ of dorsal length of head. Mouthcone 127-145 $\mu \mathrm{m}$ long, 
reaches slightly behind half of prothorax. Vertex and cheeks behind row of postocular setae with transverse sculptural lines; a few sculptural lines in central part present already from level of postocellar setae. Compound eyes with $3-4$ setae; ommatidia slightly oval, $7-10 \mu \mathrm{m}$ in diameter. Ocelli slightly oval, posterior ocelli $15-17 \mu \mathrm{m}$ long, anterior ocellus usually slightly smaller. Anteocellar setae 20-28 $\mu \mathrm{m}$ long, inserted just near compound eyes, at level of anterior ocellus; interocellar setae between posterior ocelli, in the middle of their height, $60-80$ $\mu \mathrm{m}$ long; postocellar setae very thin 7-12 $\mu \mathrm{m}$ long, located behind posterior ocelli. Postocular setae located closely to compound eyes, thin and slightly long, the longest S4 and S5 - 27-35 $\mu \mathrm{m}$ long each, seta S2 usually not formed on the whole.

Antenna (Fig. 4, Table 1) length (by extended segments) total 294-339 $\mu \mathrm{m}$, partial (segments III-VIII) 230-269 $\mu \mathrm{m}$. Segment III with slightly convex sides, 1.03-1.2 times longer than segment IV, 0.97-1.2 times longer than segment VI and 2.1-2.8 times longer than segment VIII. Segment V with nearly straight sides, most often 1.9 times as long as wide, with wide apical margin. Stylus 32-42 $\mu \mathrm{m}$ long; sense cones: $27-35 \mu \mathrm{m}$ long on segment III, 27-38 $\mu \mathrm{m}$ long on segment IV, inner cone $30-38 \mu \mathrm{m}$ long on segment VI.

Table 1. Antennal segment dimensions, female.

\begin{tabular}{|c|c|c|c|}
\hline Antennal segment & Length $[\mu \mathrm{m}]$ & Width $[\mu \mathrm{m}]$ & Length to width ratio \\
\hline I & $25-30$ & $25-33$ & $0.8-1.1$ \\
\hline II & $35-42$ & $27-31$ & $1.2-1.5$ \\
\hline III & $47-58$ & $24-28$ & $1.8-2.6$ \\
\hline IV & $42-52$ & $21-26$ & $1.8-2.2$ \\
\hline $\mathrm{V}$ & $35-43$ & $19-25$ & $1.6-2.5$ \\
\hline VI & $49-57$ & $20-22$ & $2.2-2.8$ \\
\hline VII & $12-17$ & $7-10$ & $1.3-2.0$ \\
\hline VIII & $19-25$ & $5-7$ & $3.0-4.8$ \\
\hline
\end{tabular}

Pronotum (Fig. 5) 132-145 $\mu \mathrm{m}$ long, 192-215 $\mu \mathrm{m}$ wide, 1.4-1.56 times as wide as long. Median discal area without sculpture, few sculptural lines running only along anterior and posterior margins - tenuous at anterior, stronger and irregular at posterior margin. Discal setae usually not numerous (1-15, most often 6-11), 17-27 $\mu \mathrm{m}$ long (most often 25-27 $\mu \mathrm{m}$ ). Anteroangular seta (Saa) rigid, 17-25 $\mu \mathrm{m}$ long; submarginal setae S1 (Sam) 22-38 $\mu \mathrm{m}$, S2 (Srem) 20-33 $\mu \mathrm{m}$ long. Mediolateral seta (Smlat) strong, 32-44 $\mu \mathrm{m}$ long; posteroangular setae: inner 75-108 $\mu \mathrm{m}$, outer 75-100 $\mu \mathrm{m}$ long. Posterior margin with 2 pairs of posteromarginal setae, seldom 3 pairs or 3 and 2 setae - median pair (Smpm) strong and long - 35-55 $\mu \mathrm{m}$ long, setae of second and sometimes third pair short $(17-25 \mu \mathrm{m})$ and pale (names of setae after Schliephake \& Klimt 1979).

Pterothorax - ventral: length 288-320 $\mu \mathrm{m}$, width 294-333 $\mu \mathrm{m}$, dorsal: $237-275 \mu \mathrm{m}$ long.

Mesonotum 80-108 $\mu \mathrm{m}$ long, 155-180 $\mu \mathrm{m}$ wide; sculptured with distinct, irregular, transverse, anastomosing lines turning on sides, slant towards posterior margin. Length of setae: medium (S1) 20-30 $\mu \mathrm{m}$, lateral (S2) 15-20 $\mu \mathrm{m}$, posteromarginal (S3) 17-25 $\mu \mathrm{m}$.

Metanotum (Fig. 6) 75-88 $\mu \mathrm{m}$ long, 130-150 $\mu \mathrm{m}$ wide, behind anterior margin with few transverse, slightly snakelike sculptural lines; laterally lines run longitudinally, in central area they are shallow, sparse, irregular, sometimes broken, sometimes form small, closed fields and sometimes disappear completely. Outer setae (S2) 30-40 $\mu \mathrm{m}$ long, inserted at anterior margin of notum, inner setae (S1), 32-44 $\mu \mathrm{m}$ long, moved towards the medium of sclerite 17-33 $\mu \mathrm{m}$ behind anterior margin. Campaniform sensilla absent, or - seldom - only one sensillum present. Metascutellum relatively short, 37-50 $\mu \mathrm{m}$ long, $125-148 \mu \mathrm{m}$ wide, $2.7-3.6$ times as wide as long. 

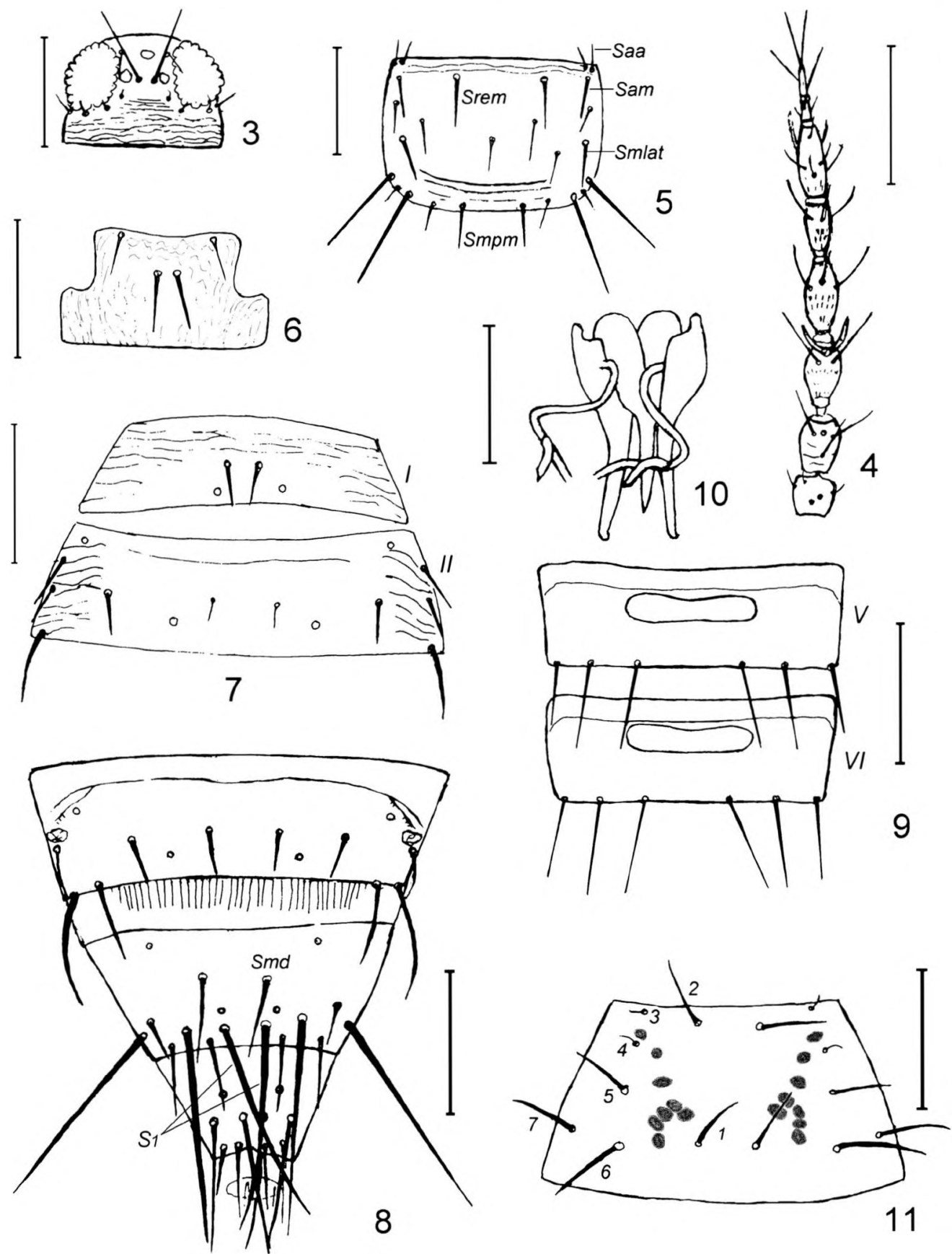

Figs 3-11. Details of morphology of Taeniothrips zurstrasseni sp. nov.; 3-8 - female, 9-10-male, 11 - larva II: 3 - head, 4 - right antenna (dorsally), 5 - pronotum (Saa - anteroangular seta, Sam - subanteromarginal seta I, Srem subanteromarginal seta II, .mlat - mediolateral seta, Smpm - medioposteromarginal seta, 6 - metanotum, 7 - abdominal tergites I and II, 8 - abdominal tergites VIII-X (Smd - mediodorsal seta, $\mathrm{S}_{1}$ - medioposteromarginal setae $\mathrm{S}_{1} ; 9$ abdominal sternites V and VI, 10 - genital apparatus (without vesicle of endotheca), 11 - pronotum. Scale bars $100 \mu \mathrm{m}$. 
Forewing 832-960 $\mu \mathrm{m}$ long, $60-73 \mu \mathrm{m}$ wide at the middle. Anterior (outer) margin straight in distal part, and only internal margin turned to apex. Wing setae relatively long and strong. Costal vein with $22-30$ setae, upper vein with $7(4+3)$ or $8(4+4)$ basal setae and 3 , or rarely 2 (exceptionally 4) distal setae. Relatively often one specimen had 3 distal setae on one wing, and 2 on the other. Of 45 individuals, 21 had 3 and 3 setae on both wings, 18 had 2 ans 3 setae, 5 had 2 and 2 and 1 had 3 and 4 setae. Wings with reduced number of distal setae, first seta placed in $+/$ - half of vein always missing. Lower vein with $8-13$ setae.

Hind tibia slender, 182-235 $\mu \mathrm{m}$ long; distal part of inner margin with a few (4-7, most often 5-6) strong, short (20-28 $\mu \mathrm{m}$ long) setae. Apical thorn most often 32-35 $\mu \mathrm{m}$ long.

Abdomen - dorsal length (extended) 1024-1184 $\mu \mathrm{m}$, width at level of segment V 333-384 $\mu \mathrm{m}$. Anterior half of tergite I sculptured all across width with transverse, irregular lines; posterior half with lines present on sides only. Tergites II-VII with short, very irregular, anastomosing sculptural lines, running laterally from seta S2. Sculpture nearly invisible on tergites VIII-X. Seta S1 on tergite I always well developed (Fig. 7), 17-38 $\mu \mathrm{m}$ long, tough (often of unequal length in the same specimen), ever distinct longer and stronger than seta S1 on following tergites. On tergites II-V, S1 most often 12-25 $\mu \mathrm{m}$ long, and up to $35 \mu \mathrm{m}$ on tergite VIII. Setae S2 on all tergites much thicker than setae S1; seta S2 22-33 $\mu \mathrm{m}$ long on tergites II-VI, 30-37 $\mu \mathrm{m}$ on tergites VII and VIII. Tergite II with three lateral setae. Hind margin of tergite VIII (Fig. 8) with complete comb, composed of dense, thin and long (up to $25 \mu \mathrm{m}$ ), but not always even, microtrichia. Laterally microtrichia short, irregularly located or absent. Segment IX 80-95 $\mu \mathrm{m}$ long. Mediodorsal seta (S.md) 56-68 $\mu \mathrm{m}$ long; setae: S1 130-152 $\mu \mathrm{m}, \mathrm{S} 2135-165 \mu \mathrm{m}$, S3 $107-$

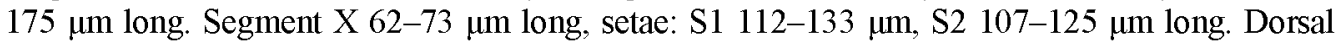
split absent, most often in the middle of hind margin small hollow with round sides present.

Sternite I with two, rarely three microsetae. Posteromarginal setae of sternites II-VI relatively thin, their length close to sternite length. Setae on sternite VII (92-115 $\mu \mathrm{m}$ long) most often a little shorter - S1 - 62-105, S2 - 85-108, S3 - 95-110 $\mu \mathrm{m}$ long; seta S1 inserted 17-30 $\mu \mathrm{m}$ before its hind margin, $\mathrm{S} 2-5-20 \mu \mathrm{m}$ before margin, S3 - directly on hind margin. Ovipositor 210-233 $\mu \mathrm{m}$ long.

Male is smaller than female, but similar to her in colour, form, sculpture of the surface and chaetotaxis. Length of body $1170-1440 \mu \mathrm{m}$.

Head 95-113 $\mu \mathrm{m}$ long, width in eyes $137-143 \mu \mathrm{m}$, in cheeks $150-157 \mu \mathrm{m}$; dorsal length of eye 55-70 $\mu \mathrm{m}$, of vertex behind the eye 32-43 $\mu \mathrm{m}$. Anteocellar seta $15-20 \mu \mathrm{m}$, interocellar 50-65 $\mu \mathrm{m}$, postocellar $-12-18 \mu \mathrm{m}$ long. Mouthcone 110-125 $\mu \mathrm{m}$ long.

Antenna - length (extended): total 100-113 $\mu \mathrm{m}$, partial 77-87 $\mu \mathrm{m}$ (Table 2). Stylus 30-35 $\mu \mathrm{m}$ long.

Table 2. Antennal segment dimensions, male

\begin{tabular}{|c|c|c|c|}
\hline Antennal segment & Length $[\mu \mathrm{m}]$ & Width $[\mu \mathrm{m}]$ & Length to width ratio \\
\hline I & $22-26$ & $24-39$ & $0.75-1.05$ \\
II & $32-38$ & $25-29$ & $1.18-1.50$ \\
III & $40-50$ & $20-25$ & $1.78-2.25$ \\
IV & $32-45$ & $16-20$ & $1.56-2.25$ \\
V & $30-38$ & $16-19$ & $1.60-2.00$ \\
VI & $41-48$ & $17-20$ & $2.13-2.57$ \\
VII & $10-15$ & $7-9$ & $1.33-1.83$ \\
VIII & $15-20$ & $5-6$ & $2.40-4.00$ \\
\hline
\end{tabular}

Pronotum 105-122 $\mu \mathrm{m}$ long, 152-185 $\mu \mathrm{m}$ wide. Length of pronotum setae in $\mu \mathrm{m}$ : Saa 15-18, Srem 20-33, Sam 22-33, Smlat 25-35, posteroangular setae: outer 50-75, inner 65-80; posteromarginal setae - 12-20, Smpm - 25-38; discal setae 15-20, number of discal setae -5-10. 
Pterothorax (ventral) 224-262 $\mu \mathrm{m}$ long, 250-281 $\mu \mathrm{m}$ wide. Mesonotum 70-85 $\mu \mathrm{m}$ long, $125-145 \mu \mathrm{m}$ wide. Setae of all three pairs of similar length $-15-23 \mu \mathrm{m}$. Metanotum $60-68 \mu \mathrm{m}$ long, $120-133 \mu \mathrm{m}$ wide. Setae S1 (20-30 $\mu \mathrm{m}$ long) located in median area of notum, $17-29 \mu \mathrm{m}$ behind fore margin. Pair of campaniform sensilla absent, seldom one sensillum present. Metascutellum 30-38 $\mu \mathrm{m}$ long, 82-113 $\mu \mathrm{m}$ wide, 2.67-3.38 times as wide as long. Forewing $620-736 \mu \mathrm{m}$ long, $47-55 \mu \mathrm{m}$ wide in the middle. Number of setae on veins: costa 17-23; upper vein - basal 7 (in one case 8), distal mostly 2, rarely 3 (of 10 individuals, 6 had 2 and 2 setae on both wings, 4 had 2 and 3 setae); lower vein 6-9. Hind tibia - 140-180 $\mu \mathrm{m}$ long. On inner side of distal part 3-5 strong, short setae, apical thorn 27-30 $\mu \mathrm{m}$ long.

Abdomen (extended) 720-896 $\mu \mathrm{m}$ long, at level of segment V 230-275 $\mu \mathrm{m}$ wide. Setae S1 on tergite I as a rule longer and stronger than on tergites II-VI, but differences in size not so striking as among females. Length of S1 on tergite I 10-28 $\mu \mathrm{m}$, on tergites II-VI 10-25 $\mu \mathrm{m}$. Setae S2 on tergites II-VIII, all of similar length, 19-28 $\mu \mathrm{m}$. Short, uneven sculptural lines with very small teeth in places present on tergite sides. Posteromarginal comb on tergite VIII complete, slightly less dense than in female, microtrichia of comb 12-20 $\mu \mathrm{m}$ long; tergite VIII lateral without microtrichia, or with only a very small number. Segment IX 57-70 $\mu \mathrm{m}$ long, 156-175 $\mu \mathrm{m}$ wide. Setae S1 25-38 $\mu \mathrm{m}, \mathrm{S} 245-58 \mu \mathrm{m}$ long. On segment X dorsal split absent.

Sternite I with two or three microsetae. Sternites III-VII with transverse, medially slightly narrowed areae porosae (Fig. 9). Their length in the middle 7-14 $\mu \mathrm{m}$, in distal, widened parts 9-20 $\mu \mathrm{m}$. Width of area porosa the largest on sternites III and IV $(80-101 \mu \mathrm{m})$; on sternite $\mathrm{V}-$ $77-98$, on VI - 65-93, on VII - 60-83 $\mu \mathrm{m}$. Aedeagus basal part light brown, distal parts of paramers narrow and much paler, with round and slightly bent up apexes. Canaliculi light (nearly colourless) and subtle, each with a single, relatively thick thorn $35-40 \mu \mathrm{m}$ long in distal part (Fig. 10).

Larva II stage. Adult larva $1.5-1.6 \mathrm{~mm}$ long.

Head 110-113 $\mu \mathrm{m}$ long, almost as wide as long. Dorsal side with long apical spot and with a few (2-4) pairs of smaller, roundish rather irregular spots along cheeks. Eye with three, less often four ommatidia. Seta D1 formed as microseta, remaining setae strong and relatively long

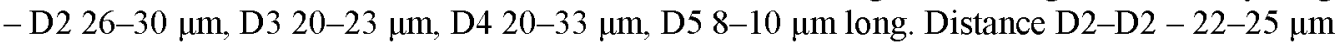
(indication of setae after Nakahara \& Vierbergen 1998).

Antenna 200-215 $\mu \mathrm{m}$ long (Table 3), dark, segments II,VI and VII darkest, segment V distinctly separated from segment IV; sense cone on segment V 20-23 $\mu \mathrm{m}$ long.

Table 3. Antennal segment dimensions, larva

\begin{tabular}{|c|c|c|c|}
\hline Antennal segment & Length $[\mu \mathrm{m}]$ & Width $[\mu \mathrm{m}]$ & Length to width ratio \\
\hline I & $20-25$ & $27-30$ & $0.7-0.9$ \\
II & $30-33$ & $25-28$ & $1.1-1.3$ \\
III & $47-53$ & $27-30$ & $1.6-1.9$ \\
IV & $38-45$ & $25-28$ & $1.4-1.8$ \\
V & $10-13$ & $20-23$ & $0.4-0.6$ \\
VI & $12-15$ & 15 & $0.8-1.0$ \\
VII & $27-30$ & $10-13$ & $2.4-3.0$ \\
\hline
\end{tabular}

Thorax and abdomen with weakly developed sculpture, plaques oblong, often nearly flat, seldom placed in rows, or only slightly snakelike transverse sculptural lines present. Plaques without microtrichia on dorsal side of thorax, on ventral side and on abdomen with very short microtrichia in some places. Setae strong and relatively long, ending bluntly or with small tear on dorsal side of body; on ventral side slightly thinner and sharp ending. Pronotum with 7 pairs of small, roundish or slightly oval spots (10-18 $\mu \mathrm{m}$ in diameter) located in two oblong rows on sides of sclerite (Fig. 11). Setae D6 and D7 the longest on pronotum, both 52-63 $\mu \mathrm{m}$ long, D3 
and D4 shortest and thinnest setae - 13-20 $\mu \mathrm{m}$ long; remaining setae - D1 37-48, D2 45-50, D5 40-45 $\mu \mathrm{m}$ long. Mesonotum with one pair of spots $25 \times 27 \mu \mathrm{m}$ in size; posterior seta (D1) stronger and longer (37-45 $\mu \mathrm{m}$ long) than anterior seta (D2) $-27-30 \mu \mathrm{m}$ long. Metanotum with one pair of spots $12 \times 17 \mu \mathrm{m}$ large; posterior setae (D1) $42-50 \mu \mathrm{m}$ long, anterior setae (D2) 25-32 $\mu \mathrm{m}$ long. All sternal setae of both segments significantly thinner and paler; V1 very

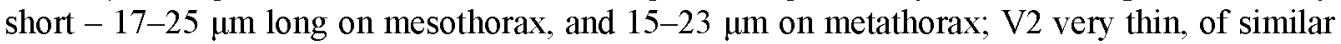
length $45-53 \mu \mathrm{m}$ on both segments. Tiles of trochanter of middle and hind legs very weakly developed, their hind margins uneven, with small, sparse teeth ending with very short microtrichia.

Table 4. Comparison of T. zurstrasseni and T. picipes according to main morphological characters.

\begin{tabular}{|c|c|c|c|c|c|}
\hline \multirow[t]{2}{*}{ No. } & Species & \multicolumn{2}{|c|}{ T. zurstrasseni sp. $\mathrm{n}$. } & \multicolumn{2}{|c|}{ T.picipes (Zetterstedt) ${ }^{1}$} \\
\hline & IMAGINES & 4599 & $13 \delta \AA$ & 4599 & $20 \pi \delta$ \\
\hline 1 & Body length & $1500-1840 \mu \mathrm{m}$ & $1170-1440 \mu \mathrm{m}$ & $1840-2240 \mu \mathrm{m}$ & $1440-1630 \mu \mathrm{m}$ \\
\hline 2 & Antennal segments III & $\begin{array}{l}47-58 \mu \mathrm{m} \text { long, } \\
2.1-2.8 \text { times } \\
\text { longer than } \\
\text { segment VIII }\end{array}$ & $\begin{array}{l}40-50 \mu \mathrm{m} \text { long, } \\
2.3-3.2 \text { times } \\
\text { longer than } \\
\text { segment VIII }\end{array}$ & $\begin{array}{l}60-75 \mu \mathrm{m} \text { long, } 3.2- \\
4.7 \text { times longer than } \\
\text { segment VIII }\end{array}$ & $\begin{array}{l}62-73 \mu \mathrm{m} \text { long, } \\
3.5-5.6 \text { times } \\
\text { longer than } \\
\text { segment VIII }\end{array}$ \\
\hline 3 & Antennal segments VIII & $19-25 \mu \mathrm{m}$ long & $15-20 \mu \mathrm{m}$ long & $15-20 \mu \mathrm{m}$ long & $12-20 \mu \mathrm{m}$ long \\
\hline 4 & $\begin{array}{l}\text { Distal setae on upper } \\
\text { vine of forewings }\end{array}$ & $\begin{array}{l}\text { mostly } 3 \& 3 \text {, more } \\
\text { rarely } 2 \& 3 \text { or } \\
2 \& 2, \text { sometimes } \\
3 \& 4\end{array}$ & $\begin{array}{l}\text { mostly } 2 \& 2 \text { or } \\
3 \& 2\end{array}$ & $\begin{array}{l}\text { mostly } 3 \& 3, \\
\text { exceptionally } 2 \& 2 \\
\text { or } 2 \& 3\end{array}$ & $\begin{array}{l}\text { mostly } 3 \& 3, \\
\text { exceptionally } 2 \& 2 \\
\text { or } 0 \& 2\end{array}$ \\
\hline 5 & $\begin{array}{l}\text { Location of setae S1 on } \\
\text { metanotum }\end{array}$ & \multicolumn{2}{|c|}{$\begin{array}{l}\text { close to middle of sclerite, } 17-33 \mathrm{~mm} \\
\text { behind fore margin of sclerite }\end{array}$} & \multicolumn{2}{|c|}{ just behind fore margin of sclerite } \\
\hline 6 & $\begin{array}{l}\text { Campaniform sensillae } \\
\text { on metanotum }\end{array}$ & \multicolumn{2}{|c|}{$\begin{array}{l}\text { usually without sensillae, sometimes } \\
\text { unpaired sensilla present }\end{array}$} & \multicolumn{2}{|c|}{$\begin{array}{l}\text { usually a pair of sensilla, sometimes with } \\
\text { only one sensillum or without sensillae }\end{array}$} \\
\hline 7 & $\begin{array}{l}\text { Length of setae S1 on } \\
\text { abdominal tergites I }\end{array}$ & $\begin{array}{l}17-38, \text { mostly } 25- \\
27 \mu \mathrm{m} \text { long, thicker } \\
\text { and longer than } \\
\text { seta } S 1 \text { on tergite II }\end{array}$ & $\begin{array}{l}10-28, \text { mostly } \\
20-22 \mu \mathrm{m} \text { long }\end{array}$ & $\begin{array}{l}7.5-27, \text { mostly } 7-12 \\
\mu m \text { long, finer and } \\
\text { shorter than seta } S 1 \\
\text { on tergite II }\end{array}$ & $\begin{array}{l}7-15, \text { mostly } 10 \\
\mu \mathrm{m} \text { long }\end{array}$ \\
\hline 8 & $\begin{array}{l}\text { Length of setae S1 on } \\
\text { abdominal tergites II }\end{array}$ & $\begin{array}{l}12-25, \text { mostly } 15- \\
17 \mu \mathrm{m} \text { long }\end{array}$ & $\begin{array}{l}12-25, \text { mostly } \\
12-15 \mu \mathrm{m} \text { long }\end{array}$ & $\begin{array}{l}20-32, \text { mostly } 22-28 \\
\mu \mathrm{m} \text { long }\end{array}$ & $\begin{array}{l}15-25, \text { mostly } 20- \\
22 \mu \mathrm{m} \text { long }\end{array}$ \\
\hline 9 & Dorsal split on segment $\mathrm{X}$ & \multicolumn{2}{|l|}{ absent } & \multicolumn{2}{|c|}{ present, relatively long } \\
\hline & 10 LAR VAE II & \multicolumn{2}{|l|}{ T. zurstrasseni sp. n. } & \multicolumn{2}{|l|}{ T. picipes (Zetterstedt) } \\
\hline 1 & $\begin{array}{l}\text { chitinization of pro-, } \\
\text { meso-, and metanotum }\end{array}$ & \multicolumn{2}{|c|}{$\begin{array}{l}\text { with } 7 \text { pairs of small, roundish spots, } \\
\text { located in rows on sides of pronotum; } \\
\text { on meso- and metanotum: distinct, } \\
\text { slightly bigger, not very regular spots, } \\
\text { one pair on each }\end{array}$} & \multicolumn{2}{|c|}{$\begin{array}{l}\text { with } 2 \text { pairs of small, roundish spots, } \\
\text { located laterally on anterior area, meso- } \\
\text { and metanotum without spots }\end{array}$} \\
\hline 2 & $\begin{array}{l}\text { antennal segments VI } \\
\text { and VII }\end{array}$ & \multicolumn{2}{|c|}{$\begin{array}{l}\text { both segments strikingly darker than } \\
\text { segments IV and V }\end{array}$} & \multicolumn{2}{|c|}{$\begin{array}{l}\text { both segments pale, as dark as or only } \\
\text { slightly darker than segments IV and V }\end{array}$} \\
\hline 3 & sculpture of body & \multicolumn{2}{|c|}{$\begin{array}{l}\text { very weakly developed, plaques } \\
\text { oblong, rarely located in rows, often } \\
\text { nearly flat, or - instead of plaques - } \\
\text { only slightly snakelike, transverse } \\
\text { sculptural lines }\end{array}$} & \multicolumn{2}{|c|}{$\begin{array}{l}\text { with plaques most often elongated, well } \\
\text { developed, located densely in transverse } \\
\text { rows, in the anterior parts of segments } \\
\text { most often ending with small microtrichiae }\end{array}$} \\
\hline 4 & $\begin{array}{l}\text { comb on hind margin of } \\
\text { segment IX }\end{array}$ & \multicolumn{2}{|c|}{$\begin{array}{l}\text { without comb - hind margin of segment } \\
\text { IX completely smooth }\end{array}$} & \multicolumn{2}{|c|}{$\begin{array}{l}\text { well developed, consisting of short, but } \\
\text { rather strong, triangular teeth on dorsal and } \\
\text { very small and weak teeth on ventral side }\end{array}$} \\
\hline
\end{tabular}

1 The T. picipes specimens required for comparison of both species were obtained from Stowinski National Park

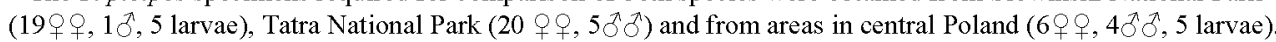


Abdominal segments with relatively long, strong and darkish tergal setae; sternal setae distinctly thinner, paler and most often shorter. For example: lengths of tergal setae on segment $\mathrm{V}$ are: D1 - 47-58, D2 - 50-52, D3 - 52-63 $\mu \mathrm{m}$; sternal setae on segment V - V1 - 20-43, V2 $-42-45$, V3 - 52-60 $\mu \mathrm{m}$ long. Abdominal tergite IX with caudal band sclerotized and pigmented from posterior margin to campaniform sensilla. Setae strong, D1 50-63, D2 67-75, D3 67-75 $\mu \mathrm{m}$ long. Hind margin of this segment without comb. Abdominal tergite $\mathrm{X}$ sclerotized and pigmented from posterior margin to $+/-$ level of campaniform sensilla, ventral to seta V4. Thin, transverse sculptural lines with very subtle teeth in fore part of segment. Setae of segment $X$ - except short and not coloured dorsolateral seta D2 (22-25 $\mu \mathrm{m}$ long) - strong and relatively long: D1 (dorsal) 50-60 $\mu \mathrm{m}$, D3 (lateral) 62-75 $\mu \mathrm{m}$, and V4 (ventral) 70-83 $\mu \mathrm{m}$ long. Segment XI on ventral side chitinous and darkened in middle part.

\section{Distinction}

Three species of the genus Taeniothrips Amyot \& Serville 1843 (zur Strassen 2003): $T$. picipes \{Zetterstedt 1828\}, T. inconsequens \{Uzel 1895\} and T. arbuti Bournier 1983) have been known so far in Europe. According to the main morphological characters T. zurstrasseni is the most similar to the first of the species mentioned above $-T$. picipes. However some essential differences can be found (Table 4). T. picipes occurs in the whole country.

\section{Ecological remarks}

On the basis of the data collected so far, one could think that Taeniothrips zurstrasseni is a resident of wetland areas and that Stachys palustris is the main (or one of the main) host for Taeniothrips zurstrasseni, at least in the study area. It also appears that the emergence and active life of the specimens of this species in the growing season starts just in June and lasts until the beginning of September. It is worth mentioning that this period coincides with the blooming period of Stachys palustris. The significance of the orchid Dactylorhiza maculata as a host plant for T. zurstrasseni in connection with its absence in the study area between 1999 and 2001 has not been explained.

\section{ACKNOWLEDGEMENTS}

I am very heartily grateful to Dr Richard zur Strassen (Forschungsinstitut Senckenberg, Frankfurt am Main) for help in resolving the taxonomic status of the new-found species. I thank Dr Halina Kucharczyk (Maria Curie-Sklodowska University in Lublin) for her kind help in preparation of the manuscript and microscopic photos of imagines and larvae of $T$. zurstrasseni. I thank Dr Wojciech Sierka (University of Silesia in Katowice) for making the microscopic photos of imagines of the new species. Acknowledgements are also due to anonymous reviewers for their valuable comments which helped me in the final preparation of this work.

\section{REFERENCES}

KuCharczyK H. 2007. Thysanoptera. In: Bogdanowicz W., Chudzicka E., Pilipipiuk I., Skibińska E. (eds), Fauna of Poland - characteristic and checklist of species. Vol. 2, pp. 391-398. Museum and Institute of Zoology, PAS, Warszawa.

NAKAHARA S. \& VIERBERGEN G. 1998. Second instar larvae of Frankliniella species in Europea Thysanoptera, Thripidae). In: VIERBERGEN G. \& TUNÇ İ. (eds), Proceedings Sixth International Symposium on Thysanoptera, Antalya, Turkey, April 27-May 1. 1998, pp. 113-120. Akdeniz University, Antalya.

PIOTROWSKA H. 1997. Podstawowe wiadomości o środowisku przyrodniczym. In: PIotrowsKA H. (ed.), Przyroda Slowińskiego Parku Narodowego, pp. 17-40. Bogucki Wydawnictwo Naukowe, Gdańsk, 320 pp.

SCHLiePHAKE G. \& KLIMT K. 1979. Thysanoptera, Fransenflügler. Die Tierwelt Deutschlands. 66. G. Fischer Verlag, Jena.

ZUR STRASSEN R. 2003. Die Terebranten Thysanopteren Europas. Die Tierwelt Deutschlands. 74. Goecke et Evears, Keltern. 
[Taeniothrips zurstrasseni (Thysanoptera, Thripidae), nowy gatunek wciornastka znaleziony w Polsce]

Nowy gatunek wciornastka Taeniothrips zurstrasseni $\mathrm{z}$ rodziny Thiripidae został znaleziony na terenie Slowińskiego Parku Narodowego, położonego w środkowej części polskiego wybrzeża Battyku, w woj. pomorskim. Osobniki dorosle i larwy zbierano z roślin rosnących na wilgotnych stanowiskach w dwóch miejscach Parku: w okolicy jeziora Dolgie Wielkie, przy brzegu kanału odwadniającego, oraz w pobliżu pólnocno-wschodniego brzegu jeziora Gardno, na lące torfowej porośniętej sitem rozpierzchlym (Juncus effusus) z domieszką różnych wilgociolubnych roślin. Najczęściej i najliczniej znajdowano osobniki Taeniothrips zurstrasseni sp. nov. na kwitnących roślinach Stachys palustris, mniej licznie (najczęściej pojedynczo) w kwiatostanach Dactylorhiza maculata, Lysimachia vulgaris, Lythrum salicaria i Linaria vulgaris.

Spośród znalezionych na terenie Europy gatunków Taeniothrips Amyot \& Serville, nowy gatunek jest morfologicznie najbardziej zbliżony do pospolitego i licznego, polifagicznego mieszkańca kwiatów Taeniothrips picipes (Zetterstedt). W pracy podano opis morfologiczny samicy, samca i larwy II stadium Taeniothrips zurstrasseni oraz omówiono cechy różniące osobniki dorosłe i larwy nowego gatunku od gatunku Taeniothrips picipes. 